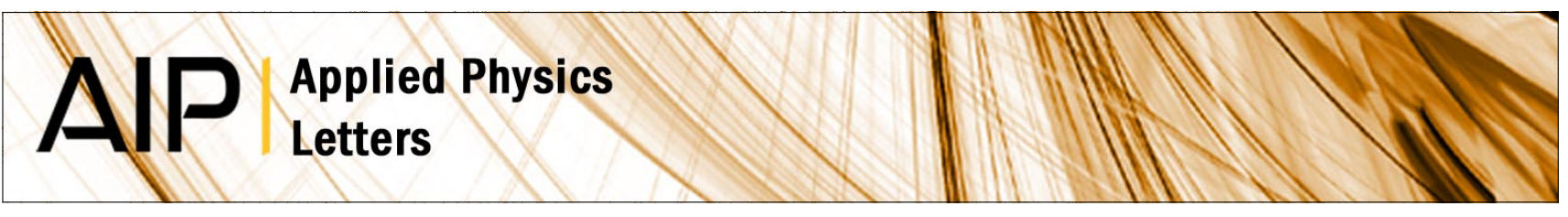

\title{
Infrared absorbance of silicene and germanene
}

Friedhelm Bechstedt, Lars Matthes, Paola Gori, and Olivia Pulci

Citation: Appl. Phys. Lett. 100, 261906 (2012); doi: 10.1063/1.4731626

View online: http://dx.doi.org/10.1063/1.4731626

View Table of Contents: http://apl.aip.org/resource/1/APPLAB/v100/i26

Published by the AIP Publishing LLC.

Additional information on Appl. Phys. Lett.

Journal Homepage: http://apl.aip.org/

Journal Information: http://apl.aip.org/about/about_the_journal

Top downloads: http://apl.aip.org/features/most_downloaded

Information for Authors: http://apl.aip.org/authors

\section{ADVERTISEMENT}

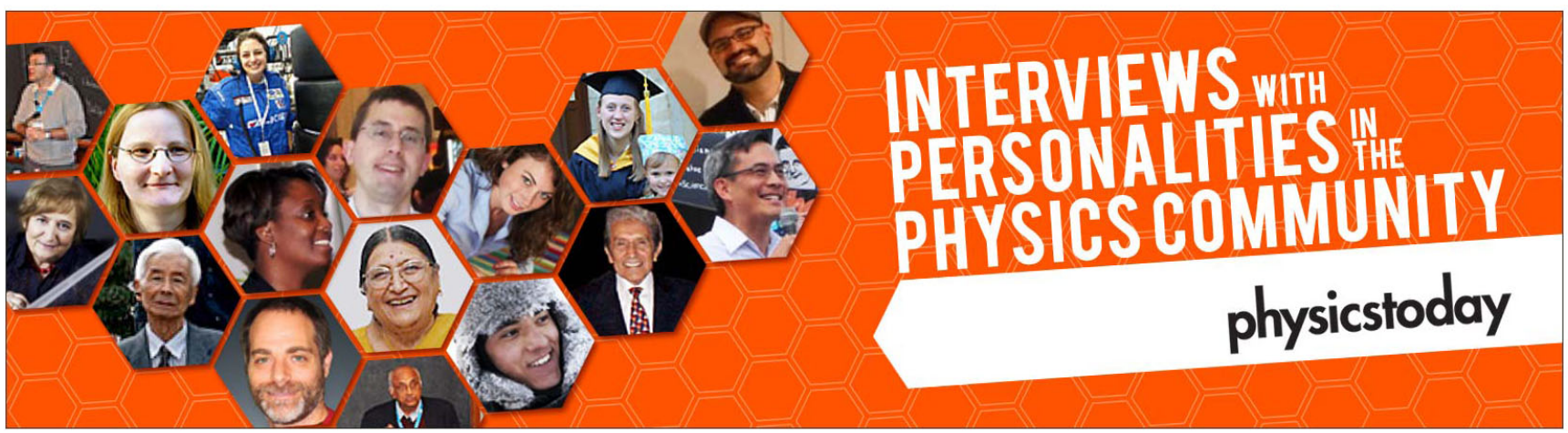




\title{
Infrared absorbance of silicene and germanene
}

\author{
Friedhelm Bechstedt, ${ }^{1}$ Lars Matthes, ${ }^{1,2}$ Paola Gori, ${ }^{3}$ and Olivia Pulci ${ }^{2}$ \\ ${ }^{1}$ Institut für Festkörpertheorie und-optik, Friedrich-Schiller-Universität, Max-Wien-Platz 1, \\ 07743 Jena, Germany \\ ${ }^{2}$ ETSF and Dipartimento di Fisica, Università di Roma Tor Vergata, Via della Ricerca Scientifica 1, \\ I-00133 Rome, Italy \\ ${ }^{3}$ ETSF and CNR-ISM, Via Fosso del Cavaliere 100, I-00133 Rome, Italy
}

(Received 3 April 2012; accepted 12 June 2012; published online 27 June 2012)

\begin{abstract}
Calculating the complex dielectric function for optical interband transitions we show that the two-dimensional crystals silicene and germanene possess the same low-frequency absorbance as graphene. It is determined by the Sommerfeld finestructure constant. Deviations occur for higher frequencies when the first interband transitions outside $K$ or $K^{\prime}$ contribute. The low-frequency results are a consequence of the honeycomb geometry but do not depend on the group-IV atom, the sheet buckling, and the orbital hybridization. The two-dimensional crystals may be useful as absorption normals in silicon technology. (C) 2012 American Institute of Physics. [http://dx.doi.org/10.1063/1.4731626]
\end{abstract}

The two-dimensional (2D) material graphene with its honeycomb crystal structure, the characteristic $\pi$-bonding, and its peculiar band structure ${ }^{1}$ has lead to the discovery of massless charge carriers, the Dirac fermions. ${ }^{2,3}$ Among the unique properties of graphene, the optical ones are of special interest. A constant infrared dynamic conductivity and optical absorbance have been predicted or measured. ${ }^{4-8}$ It has also been demonstrated experimentally that visual transparency of graphene is only determined by the Sommerfeld finestructure constant $\alpha=1 / 137.036 .^{6,7}$ The opacity or absorbance of an individual graphene sheet is given by $\pi \alpha$ in the low-frequency limit. This result is in agreement with the theory of non-interacting isotropic Dirac fermions with pseudospin (see Refs. 6, 9, 10, and references therein).

Very recently the existence of graphene-like 2D hexagonal silicon, called silicene, ${ }^{11}$ has been experimentally demonstrated, at least in form of epitaxial sheets ${ }^{12}$ and nanoribbons ${ }^{13}$ on silver surfaces. The planar hexagonal silicon may be a breakthrough for the Si technology. Theory also predicts $2 \mathrm{D}$ honeycomb structures for germanium, the so-called germanene,${ }^{14}$ and for $\mathrm{SiC} .{ }^{15}$ The $\mathrm{Si}$ - and Ge-based crystals are buckled and do not show an $s p^{2}$ hybridization but instead a mixed $s p^{2}-s p^{3}$ one. Despite a resulting buckling amplitude of $\Delta \lesssim a / 2 \sqrt{6}$ with $a$ as the 2D lattice constant, the $\pi$ and $\sigma$ bands are still decoupled in silicene and germanene, and Dirac cones are formed by the $\pi$ and $\pi^{*}$ bands. ${ }^{16}$ Neither theoretical predictions nor measurements of the optical properties of these 2D group-IV crystals are available.

In this letter, we study if the direct relation of the absorbance to the finestructure constant remains conserved in the presence of real-structure effects such as the actual Fermi velocity of the Dirac fermions and anisotropic deviations from the linear wave-vector dispersion around the $K$ and $K^{\prime}$ Dirac points. The influence of the incomplete $s p^{2}$ hybridization and hence of the buckling of the honeycomb lattice is also investigated for silicene and germanene. The investigations are based on ab-initio calculations of the dielectric function.

The structural, electronic, and optical property calculations are performed in the framework of density-functional theory (DFT) within a generalized gradient approximation (GGA). ${ }^{17}$ All-electron wave functions are generated within the projector-augmented wave (PAW) method, ${ }^{18}$ which allows for highly accurate optical transition matrix elements. ${ }^{19}$ The low-energy limit of the absorbance is calculated using a refined, non-uniform mesh of k-points around the $K$ and $K^{\prime}$ points. Explicitly, we use the DFT implementation in the Vienna $A b$-initio Simulation Package (VASP) ${ }^{20}$ The wave functions between the PAW spheres are expanded into plane waves until an energy cutoff of $500 \mathrm{eV}$. The sheets are modeled by honeycomb crystals of group-IV atoms that are arranged in superlattices with their distance of $20 \AA$. After structural optimization, the Hellmann-Feynman forces are smaller than $1 \mathrm{meV} / \AA$. In Table I results of the total energy optimizations are listed. Including graphene, clear chemical trends are visible along the row $\mathrm{C}, \mathrm{Si}$, and $\mathrm{Ge}$. The results are in agreement with other recently reported calculations. ${ }^{21,22}$

In the electronic-structure and optical studies, selfenergy and excitonic effects are not taken into account. It has been shown for graphene that in the energy range below $2 \mathrm{eV}$, where the approximation of Dirac particles is valid, the influence of the many-particle effects is negligible. ${ }^{8}$ Therefore, the Fermi velocities in Table I represent values which are only slightly smaller than velocities measured for graphene ${ }^{3}$ and silicene. ${ }^{12}$ In the range of higher photon energies, a partial compensation of the many-body effects takes place. While the quasiparticle self-energy effects give rise to a blueshift of the transition energies, the screened attraction of electrons and holes leads to a redshift.

For normal incidence, the optical properties of the 2D crystals are derived from the in-plane dielectric function

TABLE I. In-plane nearest-neighbor distance $d$ and buckling amplitude $\Delta$ for group-IV honeycomb crystals. The Fermi velocity $v_{F}$ is also listed.

\begin{tabular}{llll}
\hline \hline & $\mathrm{C}$ & $\mathrm{Si}$ & $\mathrm{Ge}$ \\
\hline$d(\AA)$ & 1.424 & 2.232 & 2.341 \\
$\Delta(\AA)$ & 0.00 & 0.45 & 0.69 \\
$v_{F}\left(10^{6} \mathrm{~m} / \mathrm{s}\right)$ & 0.829 & 0.532 & 0.517 \\
\hline \hline
\end{tabular}


$\epsilon(\omega)$ in the independent-particle approximation using Fermi's golden rule for the used superlattice arrangement. Here, we study undoped 2D group-IV crystals where the Fermi energy crosses the zero gap. Therefore, in the lowtemperature limit, only optical interband transitions take place. The Bloch bands with the full wave-vector dispersion are taken into account. For normal incidence and in-plane light polarization, the optical absorption in such a system is determined by the imaginary part of the dielectric function ${ }^{23}$

$$
\begin{aligned}
\operatorname{Im} \epsilon(\omega)= & \frac{8 \pi^{2}}{L \cdot A}\left(\frac{e}{m \omega}\right)^{2} \sum_{c, v} \sum_{\mathbf{k}}\left|M_{c v}(\mathbf{k})\right|^{2} \\
& \times \delta\left(\varepsilon_{c}(\mathbf{k})-\varepsilon_{v}(\mathbf{k})-\hbar \omega\right)
\end{aligned}
$$

with $A$ as the sheet area, $L$ the distance between the sheets in the periodic supercell arrangement, and $M_{c v}(\mathbf{k})$ the optical dipole matrix element. The optical absorption in an isolated sheet is given by the imaginary part of the $2 \mathrm{D}$ electronic polarizability $\operatorname{Im} \alpha(\omega)=L \cdot \operatorname{Im}[\epsilon(\omega)-1] / 4 \pi=L \cdot \operatorname{Im} \epsilon(\omega) / 4 \pi$ which is directly related to the thickness-independent absorbance of such a sheet $A(\omega)=\frac{4 \pi \omega}{c} \operatorname{Im} \alpha(\omega)$ with $c$ as velocity of light, i.e., ${ }^{8}$

$$
A(\omega)=\frac{\omega}{c} L \cdot \operatorname{Im} \epsilon(\omega)
$$

The frequency dependence of the ab-initio numerically calculated absorbance is plotted in Fig. 1 in a wide range of photon energies for the three studied 2D crystals.

First, we focus on the infrared absorbance. Indeed, for graphene it is known from measurements ${ }^{6,7}$ and from the theoretical predictions ${ }^{6}$ assuming Dirac fermions, that in the limit $\omega \rightarrow 0$ the absorbance approaches to $A(0)=\pi \alpha$ $(=0.022925)$ with $\alpha=e^{2} / \hbar c=1 / 137.036$. We find that this holds also for silicene and germanene and that the numerical values are $A(0)=0.02293$ (graphene), 0.02290 (silicene), and 0.02292 (germanene) in excellent agreement with the predicted value and also in good agreement with the experimental findings for roughly undoped graphene. ${ }^{6,7}$ Hence, the absorbance $A(0)$ is independent of the studied group-IV ma-

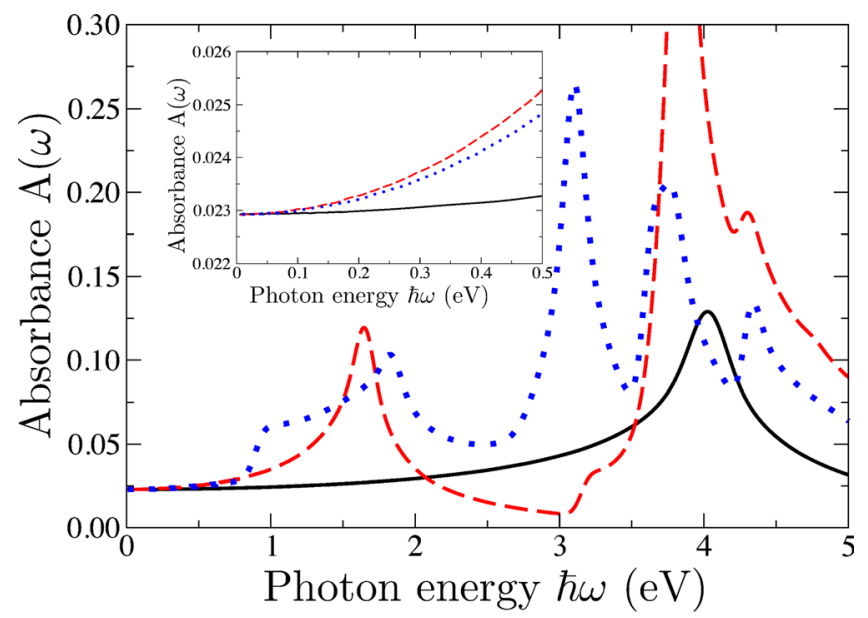

FIG. 1. Ab-initio calculated optical absorbance of graphene (black solid line), silicene (red dashed line), and germanene (blue dotted line) vs photon energy. The infrared absorbance is depicted in the inset.

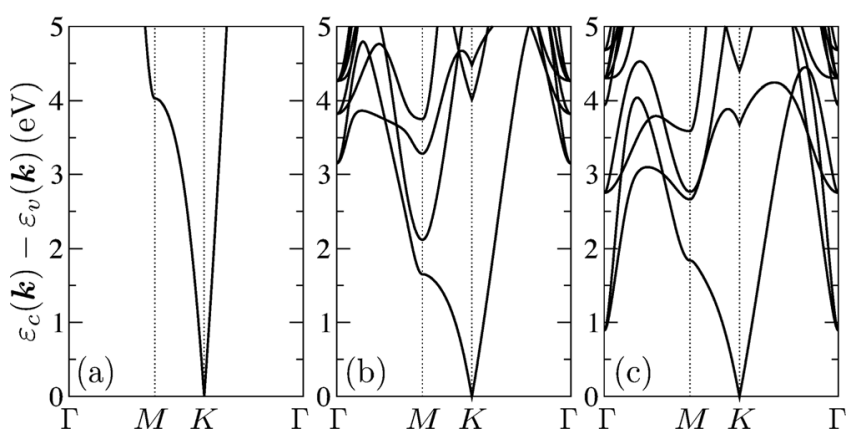

FIG. 2. Interband transition energies along high-symmetry lines in the Brillouin zone (BZ) for graphene (a), silicene (b), and germanene (c).

terial and of the sheet buckling, i.e., of the strong deviations from the $s p^{2}$ hybridization in silicene and germanene. The reason is that the point-group symmetry of a $2 \mathrm{D}$ honeycomb lattice is conserved independently of the buckling amount.

The result in Fig. 1 has been computed within the independent-particle approximation with optical interband transitions between occupied and empty Bloch states, i.e., massive Bloch particles. Of course, for low frequencies important ingredients are the linear $\mathbf{k}$ dispersion of the interband energies $\Delta \epsilon_{c v}(\mathbf{k})=\varepsilon_{c}(\mathbf{k})-\varepsilon_{v}(\mathbf{k})$ plotted in Fig. 2. The universal result in Fig. 1 for extremely small photon energies is mainly a consequence of the universal interband matrix element of the dipole operator near the Dirac points. Indeed, the average value $\left|M_{c v}(\mathbf{k})\right|^{2}=\frac{1}{2} m^{2} v_{F}^{2}$ of the optical matrix elements between pure $\pi$ and $\pi^{*}$ bands at the corner points of the $\mathrm{BZ}, \mathbf{k} \hat{=} K$ or $K^{\prime}$, is independent of the $2 \mathrm{D}$ material as shown in Fig. 3 for in-plane light polarization.

With this value of the dipole matrix elements at a $K$ or $K^{\prime}$ point it follows from Eq. (1) in the vanishing frequency limit and accounting correctly for the number of corner points of the BZ

$$
A(\omega)=2 \frac{\hbar v_{F}^{2}}{\omega} \alpha \int d^{2}(\Delta \mathbf{k}) \delta\left(2 \hbar v_{F}|\Delta \mathbf{k}|-\hbar \omega\right)
$$

and finally

$$
A(\omega)=2 \frac{\hbar v_{F}^{2}}{\omega} \alpha \frac{\pi \omega}{2 \hbar v_{F}^{2}}=\pi \alpha
$$

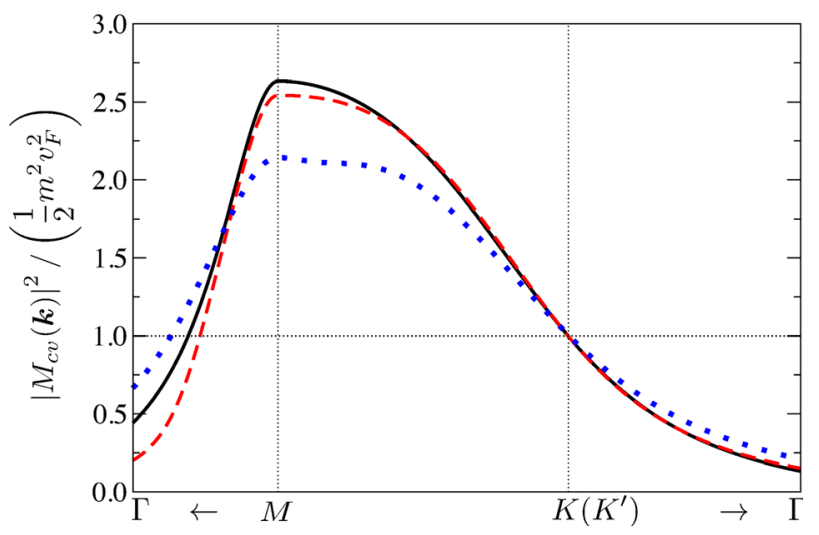

FIG. 3. Transition matrix elements of the pure $\pi-\pi^{*}$ transition along highsymmetry lines in the BZ for graphene (black solid line), silicene (red dashed line), and germanene (blue dotted line). The longitudinal gauge (Ref. 19) has been used in the calculations. 
In the limit of vanishing optical transition energies, the crystal-material dependence in the matrix elements and that in the interband energies compensate each other.

Above $\omega=0$, the absorbance increases as $\sim \omega^{2}$ in Fig. 1, as can be also shown analytically. We find that this trend is mainly a consequence of the deviation of the higher interband energies from the linearity of the Dirac cone (see Fig. 2). The wave-vector variation of the interband matrix elements in Fig. 3 around a $K$ or $K^{\prime}$ point does not lead, instead, to a significantly different frequency variation but strengthens the absorbance with rising frequency in Fig. 1.

The dipole matrix elements in Fig. 3 indicate that for low photon energies the optical properties due to $\pi \rightarrow \pi^{*}$ transitions are dominated by contributions from $\mathbf{k}$ points near the BZ boundary along the $M K$ (or $M K^{\prime}$ ) line. The matrix elements possess a maximum at an $M$ point. The effects of the group-IV material and the sheet buckling are small: at $K$ and $K^{\prime}$ the momentum matrix-element squares are the same in units of $\left(m v_{F}\right)^{2}$. Even the numerical treatments yield to values $\left|M_{c v}(\mathbf{k})\right|^{2} /\left(\frac{1}{2} m^{2} v_{F}^{2}\right)=0.995$ (graphene), 0.995 (silicene), and 1.004 (germanene) very close to the value 1 . At $M$, a minor reduction happens along the row $\mathrm{C} \rightarrow \mathrm{Si} \rightarrow \mathrm{Ge}$, whereas along $K \Gamma$ and $M \Gamma$ an opposite tendency is observed in agreement with the rising interband energies (see Fig. 2).

In contrast to the behavior in the infrared spectral region, the absorbance shows completely different frequency variations for the three $2 \mathrm{D}$ honeycomb materials in the visible and ultraviolet spectral regions, as shown in Fig. 1. The main reason is related to the different band structures, especially the interband ones in Fig. 2. The van Hove singularities in the joint density of states determine the lineshape of the absorbance. The peaks and shoulders in $A(\omega)$ can be almost related to minima, maxima, or saddle points in the interband structure (Fig. 2). Because of the strong transition strength at $M$ (see matrix elements in Fig. 3), the saddle point in the difference $\varepsilon_{c}(\mathbf{k})-\varepsilon_{v}(\mathbf{k})$ of the lowest conduction band ( $\pi^{*}$-like) and highest valence band ( $\pi$-like) gives rise to a pronounced peak at $4.0 \mathrm{eV}$ (graphene), $1.6 \mathrm{eV}$ (silicene), or $1.7 \mathrm{eV}$ (germanene). Of course, the true position should be somewhat shifted to higher energies due to quasiparticle and excitonic effects, e.g., by $0.5 \mathrm{eV}$ as shown for graphene. ${ }^{8}$ Most important is, however, that the lower-lying interband van Hove singularities in silicene and germanene do not influence the infrared absorbance at $\omega=0$. Only the absorbance increase with frequency (see inset of Fig. 1) is enhanced.

Summarizing, we have studied the optical absorbance of the $2 \mathrm{D}$ honeycomb crystals graphene, silicene, and germanene by ab-initio calculations within the independent-particle approach. We started with the full electronic structure of the crystal sheets. In the limit of vanishing frequencies, we found $A(0)=\pi \alpha$ as predicted for massless Dirac fermions. This result is explained by an isotropic linear band structure around the six $K$ and $K^{\prime}$ Dirac points and optical interband matrix elements at these points which can be universally related to the
Fermi velocity $v_{F}$ of the 2D material, i.e., to the slope of the linear bands. This result is universal for all group-IV crystals independent of the value of $v_{F}$, the degree of $s p^{2}$ and $s p^{3}$ hybridization, and the sheet buckling. For higher frequencies, the absorbance spectra start to deviate significantly with the group-IV material. The universal result $A(0)=\pi \alpha$ is suggested to use as absorption normal, especially in the terahertz regime, in silicon technology if there group-IV honeycomb layers are introduced.

We thank R. Del Sole, F. Furthmüller, K. Hannewald, and G. LeLay for valuable scientific discussions. L.M. acknowledges financial support within the EU FP7 Clermont4 Project (GA 235114). CPU time was granted from CINECA and ENEA-CRESCO. O.P. acknowledges EU FP7 SIMTECH (GA 246937) for support. P.G. acknowledges FP7 ETSF (GA 211956, Project No. 216).

${ }^{1}$ K. S. Novoselov, A. K. Geim, S. V. Morozov, D. Jiang, Y. Zhang, S. V. Dubonos, I. V. Grigorieva, and A. A. Firsov, Science 306, 666 (2004).

${ }^{2}$ K. S. Novoselov, A. K. Geim, S. V. Morozov, D. Jiang, M. I. Katsnelson, I. V. Grigorieva, S. V. Dubonos, and A. A. Firsov, Nature (London) 438, 197 (2005).

${ }^{3}$ Y. Zhang, Y.-W. Tan, H. L. Stormer, and P. Kim, Nature (London) 438, 201 (2005).

${ }^{4}$ T. Ando, Y. Zheng, and H. Suzuura, J. Phys. Soc. Jpn. 71, 1318 (2002).

${ }^{5}$ V. P. Gusynin and S. G. Sharapov, Phys. Rev. B 73, 245411 (2006).

${ }^{6}$ R. R. Nair, P. Blake, A. N. Grigorenko, K. S. Novoselov, T. J. Booth, T. Stauber, N. M. R. Peres, and A. K. Geim, Science 320, 1308 (2008).

${ }^{7}$ K. F. Mak, M. Y. Sfeir, Y. Wu, C. H. Lui, J. A. Misewich, and T. F. Heinz, Phys. Rev. Lett. 101, 196405 (2008).

${ }^{8}$ L. Yang, J. Deslippe, C.-H. Park, M. L. Cohen, and S. G. Louie, Phys. Rev. Lett. 103, 186802 (2009).

${ }^{9}$ V. Gusynin, S. Sharapov, and J. Carbotte, Int. J. Mod. Phys. B 21, 4611 (2007).

${ }^{10}$ T. Stauber, N. M. R. Peres, and A. K. Geim, Phys. Rev. B 78, 085432 (2008).

${ }^{11}$ G. G. Guzmán-Verri and L. C. Lew Yan Voon, Phys. Rev. B 76, 075131 (2007).

${ }^{12}$ P. Vogt, P. De Padova, C. Quaresima, J. Avila, E. Frantzeskakis, M. C. Asensio, A. Resta, B. Ealet, and G. Le Lay, Phys. Rev. Lett. 108, 155501 (2012).

${ }^{13}$ P. De Padova, C. Quaresima, C. Ottaviani, P. M. Sheverdyaeva, P. Moras, C. Carbone, D. Topwal, B. Olivieri, A. Kara, H. Oughaddou, B. Aufray, and G. Le Lay, Appl. Phys. Lett. 96, 261905 (2010).

${ }^{14}$ S. Cahangirov, M. Topsakal, E. Aktürk, H. Şahin, and S. Ciraci, Phys. Rev. Lett. 102, 236804 (2009).

${ }^{15}$ P. Gori, O. Pulci, M. Marsili, and F. Bechstedt, Appl. Phys. Lett. 100, 043110 (2012).

${ }^{16}$ A. Grüneis, R. Saito, G. G. Samsonidze, T. Kimura, M. A. Pimenta, A. Jorio, A. G. S. Filho, G. Dresselhaus, and M. S. Dresselhaus, Phys. Rev. B 67, 165402 (2003).

${ }^{17}$ J. P. Perdew, in Electronic Structure of Solids' 91 , edited by P. Ziesche and H. Eschrig (Akademie-Verlag, Berlin, 1991), p. 11.

${ }^{18}$ G. Kresse and D. Joubert, Phys. Rev. B 59, 1758 (1999).

${ }^{19}$ M. Gajdoš, K. Hummer, G. Kresse, J. Furthmüller, and F. Bechstedt, Phys. Rev. B 73, 045112 (2006).

${ }^{20}$ G. Kresse and J. Furthmüller, Comput. Mater. Sci. 6, 15 (1996).

${ }^{21}$ L. C. Lew Yan Voon, E. Sandberg, R. S. Aga, and A. A. Farajian, Appl. Phys. Lett. 97, 163114 (2010).

${ }^{22}$ T. H. Osborn, A. A. Farajian, O. V. Pupysheva, R. S. Aga, and L. L. Y. Voon, Chem. Phys. Lett. 511, 101 (2011).

${ }^{23}$ B. Adolph, V. I. Gavrilenko, K. Tenelsen, F. Bechstedt, and R. Del Sole, Phys. Rev. B 53, 9797 (1996). 